\title{
Revisión de la periodontitis crónica: Evolución y su aplicación clínica
}

\author{
ESCUDERO-CASTAÑO N* \\ PEREA-GARCÍA MA* \\ BASCONES-MARTÍNEZ A**
}

Escudero-Castaño N, Perea-García MA, Bascones-Martínez A. Revisión de la periodontitis crónica: Evolución y su aplicación clínica. Av Periodon Implantol. 2008; 20, 1:27-37.

\begin{abstract}
RESUMEN
El objetivo de este trabajo es realizar una descripción exhaustiva de la periodontitis crónica. La periodontitis crónica está causada por infecciones mixtas producidas por un biofilm de bacterias sibgingivales. No existe un único patógeno que sea el responsable de la etiopatologia de la periodontitis. La respuesta inmune de anticuerpos a P. intermedia, B. forsythus y T. denticola tambien ha sido demostrada en este tipo de pacientes.
\end{abstract}

\section{PALABRAS CLAVE}

Periodontitis crónica, periodontitis del adulto.

Fecha de recepción: Noviembre 2007.

Fecha de aceptación: Diciembre 2007.

\section{INTRODUCCIÓN}

La enfermedad periodontal es un proceso infeccioso de la encía y del aparato de inserción adyacente, producido por diversos microorganismos que colonizan el área supra y subgingival.

Esta enfermedad, a diferencia de la gingivitis, se caracteriza por una pérdida estructural del aparato de inserción, producida por determinadas bacterias, éstas son también necesarias pero no suficientes para que se produzca la enfermedad, siendo necesaria la presencia de un hospedador susceptible.

Desde el punto de vista histológico, las características que podemos hallar son bolsas periodontales, localización de la unión epitelial apical a la línea ameloce- mentaria, una pérdida de fibras colágenas, una elevada concentración de leucocitos polimorfonucleares en la unión y bolsa epitelial, y una migración del infiltrado celular inflamatorio hacia el tejido conectivo (1).

Para clasificar una enfermedad como periodontitis crónica, debemos en primer lugar descartar una periodontitis agresiva (PA), descartando el cumplimiento de las 3 características primarias de una PA, los pacientes deben de estar clínicamente sanos, existe agregación familiar de la enfermedad y hallamos una rápida perdida de inserción y ósea.

A continuación procederemos a centrarnos en la historia, epidemiología, características, clasificación, diagnóstico, etiopatogenia y tratamiento de la periodontitis crónica.

\footnotetext{
* Licenciado/a en Odontología. Máster de Ciencias odontológicas. Máster de Periodoncia. Facultad de Odontología. Universidad Complutense de Madrid.

** Catedrático de Medicina Bucal y Periodoncia. Departamento Medicina y Cirugía Bucofacial. Facultad de Odontología. Universidad Complutense de Madrid.
} 


\section{HISTORIA}

En los últimos años la Asociación Americana de Periodoncia ha realizado diversas clasificaciones de las enfermedades periodontales, que han ido cambiando en función de nuevos conceptos sobre la enfermedad periodontal (2).

Entre diversas clasificaciones que se realizaron a lo largo de los años, cabe destacar la de Page y Schroeder en 1985, en la que describieron en un su libro de "periodontitis in man and other animals" los diferentes tipos de periodontitis.

En la tabla 1 resumimos sus características de lo que consideraban como periodontitis del adulto.

En 1989 se reúnen un grupo de especialistas en el tema para llegar a un consenso sobre las clasificaciones de la enfermedad periodontal, tomando como referencia la de Page y Schroeder; y a través del World Workshop de 1989, se denominó Periodontitis de Adulto a aquel paciente que presentaba enfermedad periodontal a partir de los 35 años, con una tasa de progresión de la reabsorción ósea lenta y predominantemente horizontal (1) La base de las diferencias entre esta clasificación se establece principalmente en función de: La edad de aparición de la enfermedad, la tasa de progresión y la introducción o supresión de categorías. Unos años más tarde, en Europa se llega al consenso de otra nueva clasificación en el European Workshop de 1993, en el que la periodontitis del adulto está descrita como un factor primario, y no es hasta el World Workshop de 1999 que se sustituye por el nombre de periodontitis crónica; ya que la evidencia epidemiológica hallada hasta ese momento había puesto de

\section{TABLA 1.- PERIODONTITIS DEL ADULTO}

- Rango de edad > 35 años.

- Depósitos microbiológicos proporcionados con la cantidad de destrucción periodontal.

- Patrón pérdida ósea generalizado o localizado, sin evidencia de progresión rápida

- No predisposición enfermedades sistémicas

- No anomalías leucocitarias

- Flora: Gram negativos $\rightarrow$ B. melaninogenicus ss. Intermedius, E. corrodens, espiroquetas, Pg y Aa.

- Puede aparecer manifestaciones inflamatorias como fibrosis. manifiesto la posibilidad de afectación en individuos de cualquier grupo de edad e, incluso, en dentición primaria y, a pesar de su ritmo generalmente lento de progresión, algunos individuos presentaban periodos cortos de exacerbación. El término crónico es menos específico pero no debe ser interpretado como no curable.

En el siguiente cuadro esquematizaremos la evolución de la periodontitis crónica (Tabla 2).

\section{EPIDEMIOLOGÍA}

Existen pocos estudios epidemiológicos sobre la prevalencia de periodontitis crónica propiamente dicha, debido al cambio de nomenclatura realizada en el World Workshop de 1999.

En estas últimas décadas, se han realizado diversos estudios sobre la prevalencia, características de especificidad por el sitio de enfermedad periodontal reve-

\begin{tabular}{|c|}
\hline TABLA 2 \\
\hline $\begin{array}{c}\text { WORLD WORISHOP } 1989 \\
\text { Periodontitis del adulto }\end{array}$ \\
\hline
\end{tabular}

Edad $\geq 35$ años.

Tasa progresión lenta.

P. reabsorción horizontal.

Import: F.local y ambiental

Ausencia etiología sistémica.

\section{EUROPEAN WORIKSHOP 1993 \\ Periodontitis del adulto}

Descriptores primarios:

Periodontitis del adulto.

Descriptores secundarios:

Distribución dentición, ritmo de progresión, respuesta tto. micro,...

\section{WORLD WORIKSHOP 1999 \\ Periodontitis crónica}

Pérdida soporte diente.

P. progresión moderados + periodos rápida progresión.

Destrucción en $\mathrm{f}(\mathrm{x})$ factores locales.

Clasificación: extensión y severidad. 
lando detalles concernientes a la extensión en la dentición y la severidad de los defectos; evaluándolos mediante exámenes clínicos de los tejidos periodontales, radiográficos o una combinación de ambos.

Los principales estudios transversales (3-6) indican que las formas severas de periodontitis afectan a una minoría de sujetos en los países industrializados, que aumenta con el envejecimiento y que alcanza su pico a los 50-60 años.

Albandar et al en 1999 (6) muestran claramente que las bolsas más profundas y pérdida de inserción avanzada fue más pronunciada en hispanos y negros que en los blancos no hispánicos, llegando a la conclusión, que la prevalencia de enfermedad periodontal no se distribuye de manera uniforme entre las diversas razas, etnias o grupos socioeconómicos.

Debido a que la epidemiología puede cambiar en función de la población estudiada:

\section{- En Europa}

Linde et al en 1989, Papapanou et al en 1996 y Sherman et al en 2000 (7-9) demuestran en sus estudios que son pocos los sujetos que, en cada grupo de edad, sufren destrucción periodontal avanzada y sólo pocas localizaciones presentan destrucción extensa en un periodo determinado de observación. Sólo en un $10 \%$ de la enfermedad periodontal severa se presentan bolsas mayores de 5,5 mm y en comparación con datos históricos se observa un notable descenso de bolsas mayores de $4 \mathrm{~mm}$ desde 1976 en Europa

Los europeos de 35-44 años con bolsas de 3,5-5,5 $\mathrm{mm}$ (moderada) es del 13 y del $54 \%$ respectivamente. Con ligeras diferencias entre el este de Europa $(45 \%)$ y oeste de Europa (36\%)

\section{- En España}

Al analizar los resultados de la encuesta de salud oral en España del año 2000 se demostró que un $55 \%$ de adolescentes no presenta ni sangrado ni cálculo y que la prevalencia de enfermedad severa en adultos jóvenes es de sólo 4,2\%. Mientras que el grupo de 65 a 74 años presenta enfermedad severa en un $8.7 \%$.

\section{- En EEUU}

Brown et al en 1996 (10), determinó que el 15\% de adultos eran sanos, los porcentajes de gingivitis llegaban al 50\%, sólo el 33\% presentaba pérdidas de inserción de hasta $5 \mathrm{~mm}$, y un $8 \%$ de casos mostra- ba periodontitis avanzada, (con pérdidas de 6 o más $\mathrm{mm}$ de inserción), mientras que un $4 \%$ se encontraban en fase terminal.

Albandar y Rams, en el año 2002 (11), muestra que la periodontitis crónica es la forma mas frecuente de periodontitis, la prevalencia y severidad aumentan con la edad, y que las formas severas afectan únicamente a un pequeño porcentaje de la población.

\section{CARACTERÍSTICAS}

La periodontitis crónica tiene mayor prevalencia en adultos, aunque se pueden aparecer en individuos de cualquier grupo de edad, es decir, se produce tanto en la $1^{\circ}$ como en la $2^{\circ}$ dentición.

Podemos llegar a hallar diversos signos y síntomas tales como:

- Edema.

- Eritema.

- Aumento o recesión de la encía.

- Placa o cálculo supra y subgingival.

- Factores locales que aumentan el acumulo de placa sangrado o supuración al sondaje o espontánea.

- Una mayor movilidad.

- Apiñamiento o exfoliación dental.

Todas estas circunstancias pueden afectar a un número variable de dientes en función de cada individuo, con tasas variables de progresión $(1,2,12)$.

Las características clínicas es una combinación de los siguientes signos la pérdida de nivel de inserción clínica, aumento de la profundidad de bolsa, infamación gingival y pérdida ósea radiográfica.

Valorando dos de los parámetros más importantes, profundidad de sondaje y pérdida de inserción clínica, para clasificar la periodontitis crónica, se demuestra que:

En el año 2000 se publican unos parámetros a partir de la clasificación del World Workshop de 1999, en el que otorgan a la periodontitis crónica de leve a moderada (12) las siguientes características:

- Una pérdida de inserción que no supera un tercio de la longitud radicular.

- Si el diente presentara lesión furcal, ésta no superaría la clase I. 
- La profundidad de sondaje determinada no debe ser mayor de $4 \mathrm{~mm}$ para afectaciones leves, ni mayor de $6 \mathrm{~mm}$ para clasificarlas como moderadas, es decir, las pérdidas de inserción no deben ser superiores a $4 \mathrm{~mm}$.

Y en cambio, en las periodontitis crónicas severas o avanzadas, (13) podemos observar:

- Una pérdida de inserción por encima de los 5 milímetros y superior a un tercio de la longitud radicular.

- La existencia de afectaciones furcales de grado II y/o III.

- Profundidades de sondaje superiores a 6 milímetros.

\section{CLASIFICACIÓN}

La clasificación de la periodontitis crónica e establece en función de los siguientes criterios:

\section{Extensión:}

- Localizada: La que se presenta en menos de un $30 \%$.

- Generalizada: La que muestra en más de un $30 \%$ de localizaciones afectadas.

\section{Severidad:}

- Leve: Cuando la pérdida de inserción es de 1 a 2 milímetros.

- Moderada: Cuando la pérdida de inserción es de 3 a $4 \mathrm{~mm}$.

- Severa o avanzada: Cuando la pérdida de inserción es superior a $5 \mathrm{~mm}$.

\section{DIAGNÓSTICO}

El examen del estado periodontal de un paciente incluye la valoración de una serie de pruebas diagnósticas, basadas en parámetro clínicos, como la evaluación clínica de la inflamación, el nivel de inserción (NI) y profundidad de sondaje (PS), y al parámetros radiográficos para estimar la pérdida ósea (Figuras l a 4).

Las limitaciones de este tipo de mediciones es la falta de información sobre las localizaciones que están desarrollando un proceso en actividad; ya que este tipo de pruebas solo indican la destrucción tisular acontecida.

Para diagnosticar una periodontitis crónica debemos tener en cuenta las características clínicas que se presentan en la tabla 3.

\section{ETIOPATOGENIA}

En determinadas ocasiones, la enfermedad periodontal está relacionada con el sujeto, porque a pesar de la importancia de la placa en esta enfermedad, sólo algunas personas desarrollan una destrucción avanzada, y su progresión es continua, con breves episodios de exacerbación y remisión localizados $(2,14)$. Por lo tanto, determinados individuos con defectos en su sistema inflamatorio o inmunitario pueden generar periodontitis; incluso, se podría llegar a demostrar cierta predisposición genética (15).

El término infección se emplea para referirse a la presencia y multiplicación de microorganismos en el cuer-
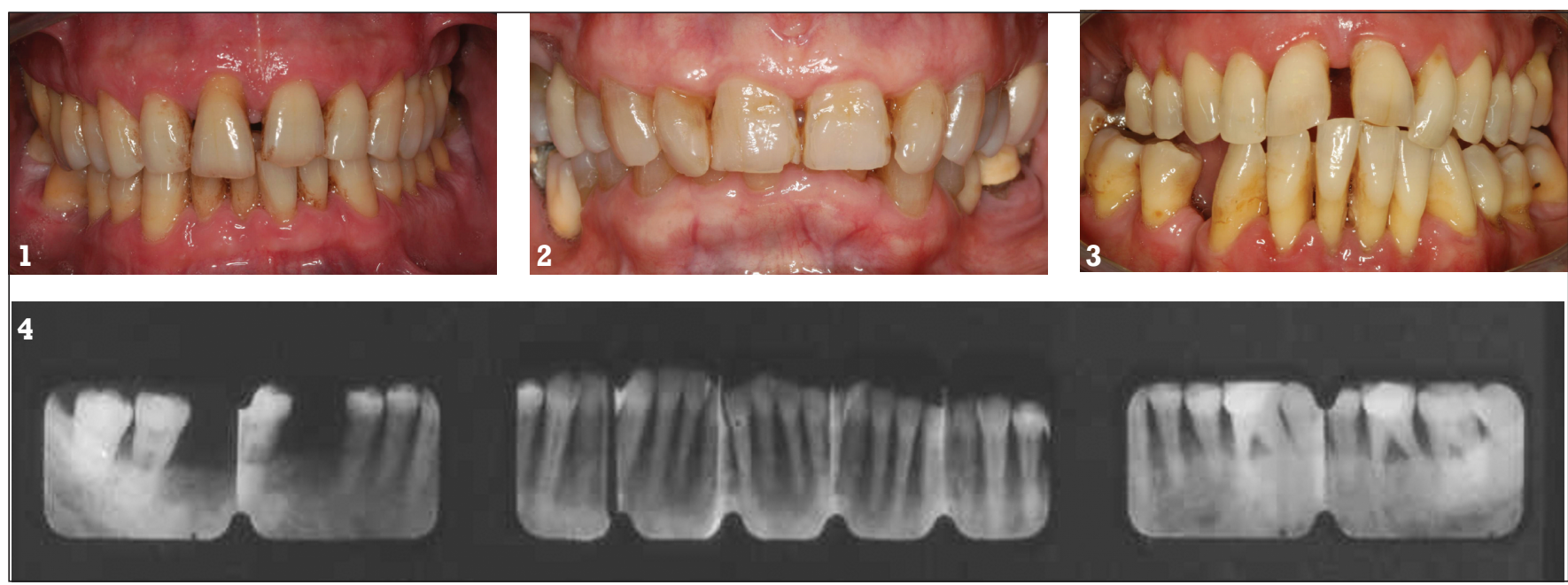

Figs.1, 2, 3 y 4. 


\section{TABLA 3.- CARACTERÍSTICAS CLÍNICAS DE LA PERIODONTITIS CRÓNICA}

- Periodontitis crónica > prevalencia en adultos, también individuos de cualquier edad.

- La magnitud de la destrucción clínica es proporcional a los niveles de higiene oral (ya que la placa inicia y sustenta este tipo de periodontitis), factores predisponentes locales (como por ejemplo, elementos retentivos de placa) y factores sistémicos de riesgo (como por ejemplo estrés, tabaquismo, enfermedades sistémicas y afectación del sistema inmune del huésped).

- La composición de la placa microbiana es variable.

- La clasificación en función de su extensión y severidad.

- La progresión sólo puede confirmarse por exámenes continuos, normalmente en localizaciones en las que el tratamiento ha sido inadecuado o nulo.

po; por lo tanto denominamos infección periodontal a la enfermedad que, localizada en encía y estructuras de soporte del diente, ligamento y hueso alveolar, está producida por un grupo determinado de bacterias provenientes de la placa subgingival que funcionan normalmente individualmente o en biofims, cuando se produce un desequilibrio entre la carga microbiana de la bolsa periodontal y los mecanismos locales y sistémicos de la respuesta del huésped. El papel que desempeñan estas bacterias en dicho desequilibrio, es el desarrollo de la periodontitis participando en la formación de la bolsa periodontal, destrucción del tejido conectivo y reabsorción del hueso alveolar a través de un mecanismo inmunopatogénico (Figura 5) (2).

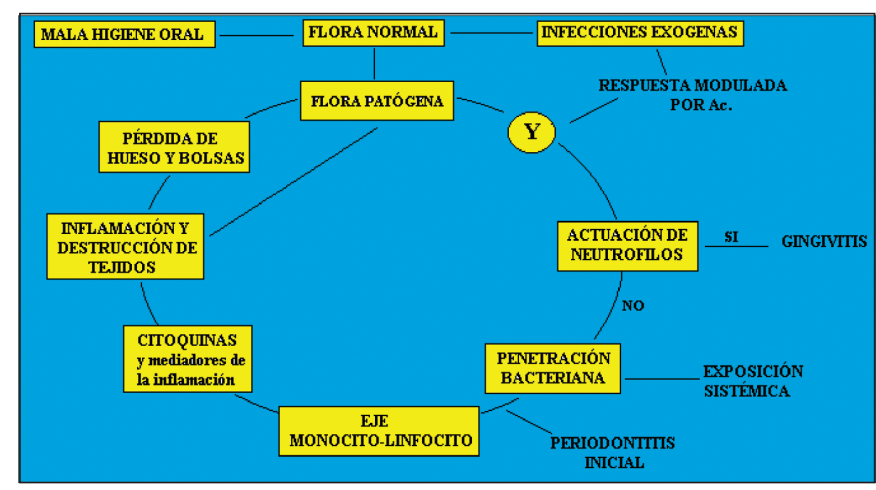

Fig.5.

Offenbacher (16) desarrolló un modelo etiopatogénico en el que la flora microbiana, al adquirir propiedades etiopatogénicas, permite al huésped ser capaz de frenar el proceso a través de las primeras líneas de defensa, con los PMN, confinando de ese modo la lesión a una gingivitis. Si ésta fracasase, la penetración bacteriana daría lugar a la activación de la segunda línea de defensa del huésped mediante el eje linfocitomonocito y la liberación de diversos tipos de citoquinas y mediadores proinflamatorios que van a producir inflamación y destrucción de los tejidos, pérdida ósea y formación de bolsas periodontales, convirtiéndose en un proceso irreversible, periodontitis, que se detendrá cuando la flora patógena se modifique en flora normal y la relación de desequilibrio se estabilice de nuevo.

El proceso destructivo de la enfermedad periodontal puede verse acelerado o ralentizado en función de diversos factores (Tabla 4).

En conclusión, cuando se desarrolla periodontitis se debe al incremento cuantitativo específico microbio-

\section{TABLA 4}

Verdaderos Factor ambiental, de comportamiento o biológico asociado confirmado factores de por una secuencia temporal en estudios longitudinales. > exposición > riesgo $\quad \%$ desarrollar enfermedad. <exposición <probabilidad adquirir enferm. Indicadores = Factores de riesgo potenciales. Factores causales, biológicamente plaude riesgo sibles, sólo demuestran asociación a periodontitis en estudios transversales y casos-controles.

Determinantes =Factores de susceptibilidad.

de riesgo Factores de riesgo no modificables.

Predictores de Factores biológicos indicativos de enfermedad, pero no forman parte riesgo. de la cadena causal de la enfermedad. Se asocian a >probabilidad de padecer la enfermedad.
EJ: tabaco y diabetes.

EJ: estrés, osteoporosis, obesidad, higiene oral.

EJ: edad, sexo, raza, genotipo, estatus socioeconómicos.

EJ: Sangrado al sondaje 
lógico o al sobrecrecimiento de especies patógenas por encima de un umbral específico, y/o provocado por la reducción de la respuesta inmune del huésped, a través de causas genéticas $(15,17,18)$, o ambientales, como son el tabaco (19), la mala higiene (3), determinada medicación inmunosupresora (20), stress (21), edad (3),... A continuación procederemos a desarrollar cada una de ellas.

\section{Papel De LAS BaCterias EN LA PERIODONTITIS}

Los microorganismos periodontales son un factor necesarios, pero no suficiente para el desarrollo de la enfermedad periodontal; por lo tanto, aunque diversas bacterias subgingivales agrupadas en biofilms son esenciales para el inicio y progresión de la enfermedad periodontal, la cantidad y el tipo no pueden explicar por si solas la severidad de la enfermedad en el adulto (22).

Existen 700 especies cultivables bacterianas de una muestra subgingival, de las cuales detectamos entre 30 y 100 en una muestra concreta. A pesar de nuestras múltiples limitaciones al: cultivarlas, tomar muestras representativas, localizar sitios enfermos con destrucción activa, las naturaleza mixta de las infecciones periodontales, el hallazgo de especies patógenas en portadores sanos,...; diversas bacterias de la placa demuestran su patogenicidad tanto en localizaciones extraorales como en animales de experimentación, produciendo una serie de productos tóxicos, como endotoxinas, amoníaco, leucotoxinas, y una serie de enzimas que producen destrucción de los tejidos periodontales.

El estudio más importante de asociaciones de bacterias o clusters lo llevo a cabo el equipo de Socransky et al (23) en el que analizó 13261 muestras de 185 pacientes evaluando 40 especies subgingivales. Los resultados describen 5 grupos:

- Grupo rojo. B. forsythus, P. gingivalis, y T. denticola. Este grupo se asociaba claramente a condiciones clínicas con mayor grado de sangrado y profundidad de bolsa.

- Grupo naranja. Con un núcleo central bastante estable, formado por P. intermedia, P. nigrescens, P. micros, F. nucleatum (subespecies vicentii, nucleatum y polymorphum) y $F$. periodonticum, y un grupo de bacterias asociadas a ellas, como Eu. nodatum, Campylobacter rectus, showae y gracilis, y St.constellatus.
Además, el grupo naranja tiene una estrecha relación con el rojo.

- Grupo amarillo. Dentro de este grupo St. mitis, oralis y sanguis tienen una muy relación muy fuerte, incluyéndose también St. gordonii, St. intermedius, y otras especies de Sterptococcus.

- Grupo verde. Con E. corrodens, Capnocytophaga gingivalis, sputigena, ochracea, Campylobacter concisus y A. actinomycetemcomitans serotipo a.

- Grupo púrpura. Solo Veillonela párvula y Actinomyces odontolyticus, muy asociados entre sí, y algo menos con los grupos naranja, verde y amarillo.

- Sin grupo. Especies sin asociaciones claras eran $A$. actinomycetemcomitans serotipo b, Actinomyces naeslundii 2 (A. viscosus), y Selenomonas noxia.

La distribución y asociaciones entre grupos demuestran la secuencia de colonización. A. viscosus y el grupo amarillo sería los colonizadores tempranos. Luego llegaría el grupo verde, especies puente para la llegada del grupo naranja, y finalmente, el grupo rojo, cuyas especies estaban ausentes en el $64 \%$ de las muestras.

De acuerdo con el World Workshop de 1996 los supuestos patógenos periodontales se dividieron en grupos de mayor o menor fuerza de evidencia:

- Evidencia fuerte: A. actinomycetemcomitans, P. gingivalis, $B$. forsythus.

- Evidencia moderada: P. intermedia/nigrescens, $C$. rectus, E. nodatum, F. nucleatum, P. micros, St. intermedius, T. denticola y espiroquetas

- Evidencia inicial. E. corrodens, bacilos entéricos, Pseudomonas sp., Selenomonas sp., Staphylococcus sp., hongos.

A pesar de que las periodontitis crónicas se asocian a un patrón microbiológico variable, autores como Mombelli et al en el 2002 (24) han tratado de detectar, a través de un trabajo de revisión, la diferencia bacteriana entre periodontitis crónicas y agresivas a través del estudio de la presencia de a.a, p.g, p.i, b.f y C.r. Hallando que: A pesar que la variante leucotóxica de A.a es la única asociada con periodontitis agresiva, ésta no se detecta en la mayoría de los sujetos diagnosticados con periodontitis agresivas. Por lo tanto, concluyó que la presencia o ausencia de los cinco patógenos estudiados no puede discriminar entre periodontitis crónicas y agresivas (aunque debemos tener en cuenta la metodología tan heterogénea entre los estudios). 


\section{Papel de la respuesta indune}

Se ha demostrado a través del estudio de Ebersole y Taubman en 1994 (25) que los individuos con periodontitis tienen niveles elevados de anticuerpos séricos frene agentes bacterianos específicos, por lo tanto el huésped desarrolla una respuesta inmunológica contra las bacterias periodontales. Demostrando una posible asociación entre esa respuesta y la naturaleza de la periodontitis.

En los estudios de Trombelli et al (26) se valora la diferencia de respuestas que puede tener diversos individuos al ataque bacteriano; planteando la posibilidad de que haya individuos con alta respuesta e individuos con baja respuesta, por lo que la aparición del cuadro sería independiente de la composición cualitativa y cuantitativa de la placa, es decir, sería el resultado de la capacidad de defensa de cada paciente. Existen individuos con baja o alta capacidad de repuesta. Pero dicha capacidad, se verá también afectada por otros factores tanto genéticos como ambientales.

\section{Papel de los factores genéticos}

A través de diversos estudios, podemos comprobar que existe una evidencia de susceptibilidad genética en la enfermedad periodontal $(15,17,18)$.

Van der Velden et al en 1993 (27) demostró el efecto de la relación fraternal en el hallazgo de un efecto significativo sobre la placa, calculo, pérdida de inserción, Pg en la encía y saliva y Pi en saliva, sin que hubiese una correlación significativa entre ellos, por lo tanto demuestra una posible relación de trasfondo genético de la periodontitis.

Michalowicz et al en el año 2000 (15) concluye que el $38 \%$ y $82 \%$ de la variabilidad del NI, IP, PS e IG se atribuye a factores genéticos; y afirma que la periodontitis crónica tiene un $50 \%$ de heredabilidad que no se altera tras ajustar con variables medioambientales como el tabaco".

Los polimorfismos son genes modificadores de la enfermedad, los sujetos heterocigóticos u homocigóticos para un gen modificador de la enfermedad pueden no necesariamente desarrollar la enfermedad; ya que también son necesarios otros factores genéticos de riesgo, como las interacciones gen-gen y/o factores de riesgo medioambientales, como las interacciones gen-ambientales. La prevalencia del polimorfismo es alrededor de un $1 \%$.
Mientras que en el estudio de Wohlfahrt et al (28) ninguno de los polimorfismos estudiados se asoció con periodontitis crónica en blancos Norteamericanos, otros estudios como el de Babel et al de 2006 (29) confirman que los polimorfismos causantes de la variabilidad en la secreción de TGF-bl e IL-6 podrían tener papel en la susceptibilidad de la P. crónica.

\section{Papel de los factores ambientales y SISTÉMICOS}

\section{TABACO}

El uso del tabaco esta relacionado directamente con una gran variedad de problemas médicos, incluyendo cáncer, parto prematuro y enfermedades cardiovasculares. Ha venido a la luz también en las últimas décadas el papel del tabaco en la etiopatogenia de la Enfermedad Periodontal. Recientemente se han publicado amplias revisiones sobre este tema, determinando que el tabaco aumenta el riesgo de sufrir enfermedad periodontal $(30,31)$

Se observa un mayor número de bolsas profundas en las localizaciones linguales de los en pacientes fumadores (31).

En estudios actuales se comprueban que el paciente fumador puede estar asociado con una supresión de la función de las células B y una alteración en la supresión de inmunoglobulinas, es decir, en pacientes fumadores se produce una alteración del sistema inmune (30). Se han observado alteraciones en numerosas funciones de los leucocitos polimorfonucleares y una disminución del número de linfocitos $\mathrm{T}$ helper, importantes para la función celular B y su producción de anticuerpos. De hecho se han demostrado niveles reducidos de IgA salivales y IgG séricas y específicamente IgG2 frente a Actinobacillus actinomycetemcomitans.

El polimorfismo del gen de la IL-1 se ha asociado a la E.P. aunque la evidencia científica no es concordante debido en primer lugar a su interacción con el tabaco además de otros factores ambientales.

Existe evidencia científica que demuestra que la respuesta inflamatoria gingival es reducida en fumadores, revelando menores localizaciones con sangrado al sondaje a paridad de IP. Los efectos de vasoconstricción sistémica de la nicotina hacen especular sobre un flujo gingival reducido en fumadores. 
El aumento de la producción de citoquinas proinflamatorias (IL-4 a parte) en pacientes fumadores, añade evidencia sobre los efectos del tabaco en la E.P.

Se produce una reducción de la adhesión celular dependiendo de la cantidad de cigarrillos fumados . Además la nicotina aumenta la producción de IL-6 e IL-8 por fibroblastos del ligamento periodontal.

El tabaco es un factor determinante en la composición de la microflora en pacientes con periodontitis, seleccionando un cluster específico de patógenos compuesto por Bf, Pm, Fn y $C r$.

\section{DIABETES NO CONTROLADA}

En primer lugar definiremos la diabetes como el grupo de desórdenes metabólicos caracterizados por la presencia de hiperglucemia debido a defectos en la secreción y/o acción de insulina

Podemos englobarla como otro de los factores de riesgo modificables (32) de enfermedad periodontal ya que existe evidencia epidemiológica suficiente para afirmar que los sujetos diabéticos no sólo presentan una mayor incidencia de periodontitis que los no diabéticos, si no que además, la forma de presentación de la misma es más severa que la de los no diabéticos, aunque la extensión parece ser similar $(32,33)$. La enfermedad periodontal es más severa en adultos con diabetes (33).

La relación Diabetes Mellitus y el estado periodontal es bidireccional: La falta de control de enfermedad aumenta el riesgo de recurrencia pero el buen control de la diabetes proporciona respuestas similares que en individuos sanos por ello es un factor de riesgo que podemos modificar.

\section{ESTRÉS Y/O DEPRESIÓN}

Se propone una asociación entre el estrés emocional, la depresión y la periodontitis a través de estudios como los de Heckmann et al o Hugoson et al $(21,34)$ en los que se ha observado una mayor pérdida de inserción y ósea en individuos que sufren estrés.

El papel del estrés puede actuar tanto en la respuesta del huésped como a nivel del cambio del comportamiento del paciente: mayor consumo de tabaco, menor dedicación a la higiene y control de placa.

\section{BACTERIAS Y EL CÁLCULO SUBGINGIVALES}

Constituyen otro factor de riesgo clave de progresión de enfermedad, ya que se ha demostrado que la falta de higiene puede llegar a desarrollar enfermedad periodontal (3); nuestro tratamiento esta destinado a eliminar ambos factores. El paciente también tiene un papel importante en el control de estos factores una vez realizado el tratamiento clínico.

\section{OTROS}

Existen otros estudios en los que encuentran asociación entre el estatus socioeconómico, la obesidad, osteoporosis, maloclusión, raza, sexos, VIH y la enfermedad periodontal.

\section{TRATAMIENTO}

En relación con la periodontitis, los estudios de Rosling (35) y de Lindhe y Nyman (36), demostraron que con el tratamiento quirúrgico, y control de placa profesional cada 2 semanas se podía controlar de manera efectiva tanto la gingivitis como la periodontitis.

La Academia Americana de Periodoncia en el 20052006 aconsejó (37) el seguimiento de una serie de pautas para el tratamiento de la periodontitis crónica:

1. Tratamiento mecánico: Raspado supra y subgingival.

2. Instrucciones de higiene oral.

3. Raspado y alisado radicular.

4. Reevaluación.

5. Cirugías:

- Terapia resectiva: Cirugía a colgajo con o sin osteotomía, amputación radicular.

- Terapia regenerativa.

- Terapia mucogingival.

En la revisión bibliográfica llevada a cabo por Kaldahl et al en 1993 (38) podemos llegar a una serie de conclusiones:

- La terapia periodontal quirúrgica y no quirúrgica puede mejorar los resultados clínicos periodontales.

- Solo a corto plazo se evidencian diferencias entre la terapia quirúrgica y no quirúrgica, aunque no a largo plazo. Mejores resultados con la terapia no quirúrgica a corto plazo. 
- La cirugía ósea produce unas mayores reducciones de la PS a corto plazo.

- La cirugía sin resección ósea se obtienen mejores NI a corto y largo plazo.

- La cirugía produjo mayor perdida de sondaje en bolsas inicialmente profundas.

Mejores resultados con RAR que con higiene supragingival.

6. Estricto programa de mantenimiento.

7. Se puede optar por diagnóstico microbiológico y la prescripción de un antibiótico adecuado. Aunque existen algunos estudios en los que se demuestra la efectividad de la terapia fotoactiva en la disminución de odontopatógenos (39-42), e incluso a ser una medida alternativa a la toma de antibióti$\cos (43)$.

No nos debemos olvidar del control de una serie de factores para el control de factores ambientales, como por ejemplo de:

- Eliminación o el recontorneado de coronas u obturaciones desbordantes o sobrecontorneadas.

- Ajuste de prótesis mal adaptadas.

- Tratamiento de caries.

- Odontoplastias.

- Movimientos dentarios.

- Restauración de contactos abiertos que favorezcan la impactación de restos de comida.

- Tratamiento del trauma oclusal.

- Extracción de dientes con pronóstico imposible.

\section{ABSTRACT}

The aim of this issue is to make a exhaustive description of the chronic periodontitis. Chronic periodontitis is caused by mixed infections with the subgingival microbiota being organized as a biofilm. The antibody reponse to ha $P$. intermedia, $B$. forsythus and T. denticola have also been demonstrated in this kind of patient.

\section{KEY WORDS}

Chronic periodontitis, adult periodontal disease.

\section{BIBLIOGRAFÍA}

1. Flemmig TF. Periodontitis. Annals of periodontology/ the American Academy of Periodontology. 1999 Dec;4 (1):32-8.
2. Bascones-Martinez A F-RE. Las enfermedades periodontales como infecciones bacterianas. Av Periodon Implantol. 2005;17(3):147-56.

3. Loe H, Anerud A, Boysen H, Smith M. The natural history of periodontal disease in man. Tooth mortality rates before 40 years of age. Journal of periodontal research. 1978 Nov; 13(6):563-72.

4. Beck JD, Koch GG, Rozier RG, Tudor GE. Prevalence and risk indicators for periodontal attachment loss in a population of older community-dwelling blacks and whites. Journal of periodontology. 1990 Aug;61(8): 521-8.

5. Pajukoski H, Meurman JH, Snellman-Grohn S, Sulkava $\mathrm{R}$. Oral health in hospitalized and nonhospitalized community-dwelling elderly patients. Oral surgery, oral medicine, oral pathology, oral radiology, and endodontics. 1999 Oct;88(4):437-43.

6. Albandar JM, Brunelle JA, Kingman A. Destructive periodontal disease in adults 30 years of age and older in the United States, 1988-1994. Journal of periodontology. 1999 Jan;70(1):13-29.

7. Sherman JA, McGurk M. Lack of correlation between water hardness and salivary calculi in England. The British journal of oral \& maxillofacial surgery. 2000 Feb;38(1):50-3.

8. Papapanou PN. Periodontal diseases: epidemiology. Annals of periodontology / the American Academy of Periodontology. 1996 Nov;1(1):1-36.

9. Lindhe J, Okamoto H, Yoneyama T, Haffajee A, Socransky SS. Periodontal loser sites in untreated adult subjects. Journal of clinical periodontology. 1989 Nov;16(10): 671-8.

10. Brown LJ, Brunelle JA, Kingman A. Periodontal status in the United States, 1988-1991: prevalence, extent, and demographic variation. Journal of dental research. 1996 Feb;75 Spec No:672-83.

11. Albandar JM, Rams TE. Global epidemiology of periodontal diseases: an overview. Periodontology 2000. 2002;29:7-10.

12. Parameter on chronic periodontitis with slight to moderate loss of periodontal support. Journal of periodontology. 2000;71:853-5. 
13. Parameter on chronic periodontitis with advanced loss of periodontal support. American Academy of Periodontology. Journal of periodontology. 2000 May;71(5 Suppl):856-8.

14. Papapanou PN, Wennstrom JL, Grondahl K. Periodontal status in relation to age and tooth type. A cross-sectional radiographic study. Journal of clinical periodontology. 1988 Aug; 15(7):469-78.

15. Michalowicz BS, Diehl SR, Gunsolley JC, Sparks BS, Brooks CN, Koertge TE, et al. Evidence of a substantial genetic basis for risk of adult periodontitis. Journal of periodontology. 2000 Nov;71(11):1699-707.

16. Offenbacher S. Periodontal diseases: pathogenesis. Annals of periodontology / the American Academy of Periodontology. 1996 Nov;1(1):821-78.

17. Kinane DF, Shiba H, Hart TC. The genetic basis of periodontitis. Periodontology 2000. 2005;39:91-117.

18. Hart TC, Kornman KS. Genetic factors in the pathogenesis of periodontitis. Periodontology 2000. 1997 Jun; 14:202-15.

19. Baharin B, Palmer RM, Coward P,Wilson RF. Investigation of periodontal destruction patterns in smokers and nonsmokers. Journal of clinical periodontology. 2006 Jul;33(7):485-90.

20. Ciavarella Domenico Guiglia R. CG, Di Cosola M, Di Liberto Ch, Sabatucci A, Escudero N, Bascones A, Lo Muzio L. Actualización en sobrecrecimiento gingival producido por la Ciclosporina A en trasplantes renales. Med Oral, Patol Oral y Cir Bucal 2007;12(1): 10-6.

21. Heckmann SM, Linke JJ, Graef F, Foitzik C, Wichmann MG, Weber HP. Stress and inflammation as a detrimental combination for peri-implant bone loss. Journal of dental research. 2006 Aug;85(8):711-6.

22. Kornman KS, Newman MG, Moore DJ, Singer RE. The influence of supragingival plaque control on clinical and microbial outcomes following the use of antibiotics for the treatment of periodontitis. Journal of periodontology. 1994 Sep;65(9):848-54.

23. Socransky SS, Haffajee $A D$, Cugini MA, Smith C, Kent $\mathrm{RL}, \mathrm{Jr}$. Microbial complexes in subgingival plaque. Journal of clinical periodontology. 1998 Feb;25(2): 134-44.
24. Mombelli A, Casagni F, Madianos PN. Can presence or absence of periodontal pathogens distinguish between subjects with chronic and aggressive periodontitis? A systematic review. Journal of clinical periodontology. 2002;29 Suppl 3:10-21; discussion 37-8.

25. Ebersole JL, Taubman MA. The protective nature of host responses in periodontal diseases. Periodontology 2000. 1994 Jun;5:112-41.

26. Trombelli L, Farina R, Manfrini R, Tatakis DN. Modulation of clinical expression of plaque-induced gingivitis: effect of incisor crown form. Journal of dental research. 2004 Sep;83(9):728-31.

27. van der Velden U, Abbas F, Armand S, de Graaff J, Timmerman MF, van derWeijden GA, et al. The effect of sibling relationship on the periodontal condition. Journal of clinical periodontology. 1993 Oct;20(9):68390.

28. Wohlfahrt JC, Wu T, Hodges JS, Hinrichs JE, Michalowicz BS. No association between selected candidate gene polymorphisms and severe chronic periodontitis. Journal of periodontology. 2006 Mar;77(3):426-36.

29. Babel N, Cherepnev G, Babel D, Tropmann A, Hammer M, Volk HD, et al. Analysis of tumor necrosis factor-alpha, transforming growth factor-beta, interleukin-10, IL-6, and interferon-gamma gene polymorphisms in patients with chronic periodontitis. Journal of periodontology. 2006 Dec;77(12):1978-83.

30. Al-Ghamdi HS, Anil S. Serum antibody levels in smoker and non-smoker saudi subjects with chronic periodontitis. Journal of periodontology. 2007 Jun;78(6):1043-50.

31. Haffajee AD, Socransky SS. Relationship of cigarette smoking to the subgingival microbiota. Journal of clinical periodontology. 2001 May;28(5):377-88.

32. Genco RJ, Loe $H$. The role of systemic conditions and disorders in periodontal disease. Periodontology 2000. 1993 Jun;2:98-116.

33. Oliver RC, Tervonen T. Periodontitis and tooth loss: comparing diabetics with the general population. Journal of the American Dental Association (1939). 1993 Dec;124(12):71-6.

34. Hugoson $A$, Ljungquist $B$, Breivik T. The relationship of some negative events and psychological factors to periodontal disease in an adult Swedish population 50 to 
80 years of age. Journal of clinical periodontology. 2002 Mar;29(3):247-53.

35. Rosling B, Nyman S, Lindhe J. The effect of systematic plaque control on bone regeneration in infrabony pockets. Journal of clinical periodontology. 1976 Feb;3(1):38-53.

36. Lindhe J, Nyman S. The effect of plaque control and surgical pocket elimination on the establishment and maintenance of periodontal health. A longitudinal study of periodontal therapy in cases of advanced disease. Journal of clinical periodontology. 1975 Apr;2 (2):67-79.

37. Treatment of plaque-induced gingivitis, chronic periodontitis, and other clinical conditions. Pediatric dentistry. 2005;27(7 Reference Manual):202-11.

38. Kaldahl WB, Kalkwarf KL, Patil KD. A review of longitudinal studies that compared periodontal therapies. Journal of periodontology. 1993 Apr;64(4):243-53.

39. Wilson M. Lethal photosensitisation of oral bacteria and its potential application in the photodynamic therapy of oral infections. Photochem Photobiol Sci. 2004 May;3(5):412-8.
40. Hayek RR, Araujo NS, Gioso MA, Ferreira J, BaptistaSobrinho CA, Yamada AM, et al. Comparative study between the effects of photodynamic therapy and conventional therapy on microbial reduction in ligatureinduced peri-implantitis in dogs. Journal of periodontology. 2005 Aug;76(8):1275-81.

41. Andersen R, Loebel N, Hammond D, Wilson M. Treatment of periodontal disease by photodisinfection compared to scaling and root planing. J Clin Dent. 2007;18(2):34-8.

42. De Almeida JM, Theodoro LH, Bosco AF, Nagata MJ, Oshiiwa M, Garcia VG. Influence of photodynamic therapy on the development of ligature-induced periodontitis in rats. Journal of periodontology. 2007 Mar;78(3):566-75.

43. Komerik N, Wilson M, Poole S. The effect of photodynamic action on two virulence factors of gramnegative bacteria. Photochemistry and photobiology. 2000 Nov;72(5):676-80.

\section{CORRESPONDENCIA}

Naira Escudero Castaño

Correo electrónico: nayramadrid@hotmail.com 